\title{
THE MINIOWITZ AND VUORINEN THEOREMS \\ FOR SOME CLASS OF MAPPINGS WITH NON-BOUNDED CHARACTERISTICS
}

\author{
E. Sevost'yanov
}

November 2, 2018

\begin{abstract}
The paper is devoted to studying classes of mappings with unbounded characteristics of quasiconformality. Namely, we prove that the normal families of $Q$-mappings have the logarithmic order of growth in a neighborhood of a point. Moreover, we establish sufficient conditions for $Q$ provided normality of mappings $f: D \rightarrow \overline{\mathbb{R}^{n}}, n \geq 2$, omitting points of a set $E_{f}$ obeying $c\left(E_{f}\right) \geq \delta, \delta>0$, where $c(\cdot)$ is an appropriate set function.
\end{abstract}

2010 Mathematics Subject Classification: Primary 30C65; Secondary $30 \mathrm{C} 62$

\section{Introduction}

The paper is devoted to studying ring $Q$-mappings, which generalize $Q$-mappings, introduced in [15] (cf. [22] and [6] for homeomorphisms). The class of ring $Q$-mappings contains many known classes of mappings such as analytic functions, quasiconformal mappings, mappings with bounded and finite distortion, etc.

Throughout the paper, $m$ denotes the Lebesgue measure in $\mathbb{R}^{n}, n \geq 2, \overline{\mathbb{R}^{n}}=$ $\mathbb{R}^{n} \cup\{\infty\}$ is the one-point compactification of $\mathbb{R}^{n}$, and $M(\Gamma)$ stands for the conformal modulus of families of curves $\gamma$ in $\mathbb{R}^{n}$ (see e.g. [27, 6.I]). For $\overline{\mathbb{R}^{n}}$, we use the spherical (chordal) distance $h(x, y)=|\pi(x)-\pi(y)|$, where $\pi$ is a stereographical projection of $\overline{\mathbb{R}^{n}}$ onto the sphere $S^{n}\left(\frac{1}{2} e_{n+1}, \frac{1}{2}\right)$ in $\mathbb{R}^{n+1}$ :

$$
h(x, \infty)=\frac{1}{\sqrt{1+|x|^{2}}}, \quad h(x, y)=\frac{|x-y|}{\sqrt{1+|x|^{2}} \sqrt{1+|y|^{2}}}, \quad x \neq \infty \neq y .
$$

Given a domain $D$ and two sets $E$ and $F$ in $\overline{\mathbb{R}^{n}}, n \geq 2, \Gamma(E, F, D)$ denotes the family of all paths $\gamma:[a, b] \rightarrow \overline{\mathbb{R}^{n}}$ which join $E$ and $F$ in $D$, i.e., $\gamma(a) \in E, \gamma(b) \in F$ and $\gamma(t) \in D$ 
for $a<t<b$. We write $\Gamma(E, F)=\Gamma\left(E, F, \overline{\mathbb{R}^{n}}\right)$ when $D=\overline{\mathbb{R}^{n}}$. Let $r_{0}=\operatorname{dist}\left(x_{0}, \partial D\right)$ and $Q: D \rightarrow[0, \infty]$ be a measurable function. Set

$$
\begin{gathered}
R\left(r_{1}, r_{2}, x_{0}\right)=\left\{x \in \mathbb{R}^{n}: r_{1}<\left|x-x_{0}\right|<r_{2}\right\}, \\
S_{i}=S\left(x_{0}, r_{i}\right)=\left\{x \in \mathbb{R}^{n}:\left|x-x_{0}\right|=r_{i}\right\}, \quad i=1,2 .
\end{gathered}
$$

A mapping $f: D \rightarrow \overline{\mathbb{R}^{n}}$ is said to be a ring $Q$-mapping at a point $x_{0} \in D$ if the inequality

$$
M\left(f\left(\Gamma\left(S_{1}, S_{2}, R\right)\right)\right) \leq \int_{R} Q(x) \cdot \eta^{n}\left(\left|x-x_{0}\right|\right) d m(x)
$$

holds for any $R=R\left(r_{1}, r_{2}, x_{0}\right), 0<r_{1}<r_{2}<r_{0}$, and every measurable function $\eta:\left(r_{1}, r_{2}\right) \rightarrow[0, \infty]$ with

$$
\int_{r_{1}}^{r_{2}} \eta(r) d r \geq 1
$$

Note that for $Q(x) \leq K=$ const, the class of $Q$-mappings contains quasiregular mappings, whereas 1-mappings have within themselves conformal mappings (see [20], 21] and [19, Theorem 1]).

In [23], we establish conditions on $Q$ ensuring the equicontinuity and normality of families of mappings satisfying (1.1) and omitting points of a fixed set of positive capacity. These conditions are sufficient for equicontinuity but not necessary. In particular, a family of mappings $f: D \rightarrow \overline{\mathbb{R}^{n}} \backslash E$ obeying (1.1) is normal whenever cap $E>0$ and $Q \in F M O$ (see [23, Theorem 5.1]).

The paper consists of two main parts devoted to studying the equicontinuity property for the mappings satisfying the relations (1.1)-(1.2). In the first part we establish necessary and sufficient conditions providing the equicontinuity for a family of ring $Q$ mappings, imposing an appropriate condition on $Q$, e.g. FMO-condition. Note that our results generalize the known results by Miniowitz for quasiregular mappings, see [17, Theorem 1]. For the plane quasiregular mappings we refer to [11, Theorem 4.3.II] (cf. [30]). The main result in this part is the following

Theorem 1.1. A family of all discrete open ring $Q$-mappings $f: D \rightarrow \overline{\mathbb{R}^{n}}$ at a point $x_{0} \in D$ with $Q \in F M O\left(x_{0}\right)$ is equicontinuous at $x_{0}$ if and only if there exist $p=p(n, Q)>0, C_{n}>0$ and $\varepsilon_{0} \in\left(0, \operatorname{dist}\left(x_{0}, \partial D\right)\right)$ such that

$$
h\left(f(x), f\left(x_{0}\right)\right) \leq C_{n}\left\{\frac{1}{\log \frac{1}{\left|x-x_{0}\right|}}\right\}^{p}
$$

for every $x \in B\left(x_{0}, \varepsilon_{0}\right)$, where $B\left(x_{0}, \varepsilon\right)=\left\{x \in \mathbb{R}^{n}:\left|x-x_{0}\right|<\varepsilon\right\}$.

Following [8], we say that a function $\varphi: D \rightarrow \mathbb{R}$ has finite mean oscillation (FMO) at a point $x_{0} \in D$ if

$$
\limsup _{\varepsilon \rightarrow 0} \frac{1}{\Omega_{n} \varepsilon^{n}} \int_{B\left(x_{0}, \varepsilon\right)}\left|\varphi(x)-\widetilde{\varphi_{\varepsilon}}\right| d m(x)<\infty,
$$


where

$$
\widetilde{\varphi_{\varepsilon}}=\frac{1}{\Omega_{n} \varepsilon^{n}} \int_{B\left(x_{0}, \varepsilon\right)} \varphi(x) d m(x)
$$

is the mean of the function $\varphi(x)$ over the ball $B\left(x_{0}, \varepsilon\right)$. Note that $F M O$ is not $B M O_{l o c}$ ([15, p. 211]). It is well known [9] that $L^{\infty}(D) \subset B M O(D) \subset L_{l o c}^{p}(D)$ for all $1 \leq p<$ $\infty$, but $F M O(D) \not \subset L_{l o c}^{p}(D)$ for any $p>1$ (see [15]).

In the second part of the paper we establish sufficient conditions of the equicontinuity for mappings $f: D \rightarrow \overline{\mathbb{R}^{n}} \backslash E_{f}$ omitting the points of a set $E_{f}$, where $E_{f}$ is a set of positive capacity depending on $f$. Note that, in the general case, the condition cap $E_{f}>0$ does not imply the equicontinuity (normality) of a family of $K$-quasiregular mappings $f: D \rightarrow \overline{\mathbb{R}^{n}} \backslash E_{f}$. For example, the family $f_{m}(z)=z^{m}, z \in \mathbb{C}$, $D:=B(0,2)=\{z \in \mathbb{C}:|z|<2\}$ for $n=2$ is not normal. It is known that, if a set $E=E_{f}$ does not depend on $f$, the corresponding family of mappings is equicontinuous (see [21, Corollary 2.7.III] for bounded $Q$ and [23, Theorems 5.1-5.2] for more general $Q)$. However, in the present paper we do not require an existence of such "general" $E$. Following [28, we restrict the family $\left\{E_{f}\right\}$ involving a set function $c(\cdot)$ (see the relation (4.1) ). The restrictions of such type have been earlier applied for bounded $Q$ (cf. [28]). The following statement is one of the main results of the paper.

Theorem 1.2. Let $\mathfrak{F}_{Q, \delta}$ be a family of open discrete ring $Q$-mappings $f: D \rightarrow$ $\overline{\mathbb{R}^{n}} \backslash E_{f}$ at $x_{0} \in D$ with $c\left(E_{f}\right) \geq \delta>0$, where $E_{f}$ is compact. Assume that either of the following conditions holds: 1) $Q \in F M O\left(x_{0}\right)$; 2) $q_{x_{0}}(r) \leq C \cdot\left(\log \frac{1}{r}\right)^{n-1}$ where $C>0$ is a positive constant and $q_{x_{0}}(r)$ is the integral mean of $Q(x)$ over the sphere $S\left(x_{0}, r\right)$; 3)

$$
\int_{0}^{\varepsilon_{0}} \frac{d t}{t q_{x_{0}}^{\frac{1}{n-1}}(t)}=\infty
$$

for some $\varepsilon_{0}=\varepsilon_{0}\left(x_{0}\right)$. Then the family $\mathfrak{F}_{Q, \delta}$ is equicontinuous at $x_{0}$.

\section{Preliminaries}

Recall the needed definitions and notions (see also [23]). Let $D$ be a domain in $\mathbb{R}^{n}$, $n \geq 2$. A mapping $f: D \rightarrow \mathbb{R}^{n}$ is said to be discrete if the preimage $f^{-1}(y)$ of every point $y \in \mathbb{R}^{n}$ consists of isolated points, and open if the image of every open set $U \subset D$ is open in $\mathbb{R}^{n}$.

If $U$ is an open set in $\mathbb{R}^{n}$, we denote by $C_{0}(U)$ the class of all continuous functions in $U$ whose support is a compact subset of $U$. Following [12] and [21, a condenser is a pair $E=(A, C)$, where $A \subset \mathbb{R}^{n}$ is open and $C$ is a non-empty compact set contained in $A$. Define the quantity

$$
\operatorname{cap} E=\operatorname{cap}(A, C)=\inf _{u \in W_{0}(E)} \int_{A}|\nabla u|^{n} d m(x)
$$


which is called the capacity of the condenser $E$, where $|\nabla u|=\left(\sum_{i=1}^{n}\left(\partial_{i} u\right)^{2}\right)^{1 / 2}, W_{0}(E)=$ $W_{0}(A, C)$ is a family of nonnegative functions $u: A \rightarrow \mathbb{R}^{1}$ such that (1) $u \in C_{0}(A)$, (2) $u(x) \geq 1$ for $x \in C$ and (3) $u$ is $A C L$ (absolutely continuous on lines, see [21]).

Let $f: D \rightarrow \mathbb{R}^{n}$ be a discrete open mapping, $\beta:[a, b) \rightarrow \mathbb{R}^{n}$ be a curve and $x \in f^{-1}(\beta(a))$. A curve $\alpha:[a, c) \rightarrow D$ is called a maximal $f$-lifting of $\beta$ starting at $x$ if (1) $\quad \alpha(a)=x ;(2) \quad f \circ \alpha=\left.\beta\right|_{[a, c)} ;(3) \quad$ there exists no path $\alpha^{\prime}:\left[a, c^{\prime}\right) \rightarrow D$ such that $\alpha=\left.\alpha^{\prime}\right|_{[a, c)}$ and $f \circ \alpha^{\prime}=\left.\beta\right|_{\left[a, c^{\prime}\right)}$ whenever $c<c^{\prime} \leq b$. For a discrete open mapping $f$, every curve $\beta$ with $x \in f^{-1}(\beta(a))$ has a maximal $f$-lifting starting at a point $x$ (see [21, Corollary 3.3.II], see also [14, Lemma 3.12]).

The following statement was earlier formulated and exploited in more special cases (see [23, Lemmas 3.2-3.3]).

Lemma 2.1. Let $f: D \rightarrow \overline{\mathbb{R}^{n}}, n \geq 2$, be an open discrete ring $Q$-mapping at a point $x_{0} \in D$. Suppose that there exist numbers $\varepsilon_{0} \in\left(0\right.$, dist $\left.\left(x_{0}, \partial D\right)\right), \varepsilon_{0}^{\prime} \in\left(0, \varepsilon_{0}\right)$ and a family of nonnegative Lebesgue measurable functions $\left\{\psi_{\varepsilon}(t)\right\}, \psi_{\varepsilon}:\left(\varepsilon, \varepsilon_{0}\right) \rightarrow[0, \infty]$, $\varepsilon \in\left(0, \varepsilon_{0}^{\prime}\right)$, such that

$$
\int_{\varepsilon<\left|x-x_{0}\right|<\varepsilon_{0}} Q(x) \cdot \psi_{\varepsilon}^{n}\left(\left|x-x_{0}\right|\right) d m(x) \leq F\left(\varepsilon, \varepsilon_{0}\right) \quad \forall \varepsilon \in\left(0, \varepsilon_{0}^{\prime}\right),
$$

where $F\left(\varepsilon, \varepsilon_{0}\right)$ is a given function and

$$
0<I\left(\varepsilon, \varepsilon_{0}\right):=\int_{\varepsilon}^{\varepsilon_{0}} \psi_{\varepsilon}(t) d t<\infty \quad \forall \varepsilon \in\left(0, \varepsilon_{0}^{\prime}\right) .
$$

Then

$$
\operatorname{cap} f(E) \leq F\left(\varepsilon, \varepsilon_{0}\right) / I^{n}\left(\varepsilon, \varepsilon_{0}\right) \quad \forall \varepsilon \in\left(0, \varepsilon_{0}^{\prime}\right),
$$

where $E=(A, C), A=B\left(x_{0}, r_{0}\right), C=\overline{B\left(x_{0}, \varepsilon\right)}, r_{0}=\operatorname{dist}\left(x_{0}, \partial D\right), A:=\mathbb{R}^{n}$ whenever $D:=\mathbb{R}^{n}$.

Proof. Consider a condenser $E=(A, C)$, where $A$ and $C$ are defined as above. Obviously the pair $f(E)=(f(A), f(C))$ is also condenser. If cap $f(E)=0$, (2.3) is trivial. Thus, assume that cap $f(E) \neq 0$. One can assume that $\infty \notin f(A)$.

Let $\Gamma_{E}$ be a family of curves $\gamma:[a, b) \rightarrow A$ such that $\gamma(a) \in C$ and $|\gamma| \cap(A \backslash F) \neq \varnothing$ for every compact set $F \subset A$ where $|\gamma|=\left\{x \in \mathbb{R}^{n}: \exists t \in[a, b): \gamma(t)=x\right\}$ is a locus of $\gamma$. It is known that cap $E=M\left(\Gamma_{E}\right)$ (see, e.g. [21, Proposition 10.2.II]).

Consider a family $\Gamma_{f(E)}$ for the condenser $f(E)$. Note that every $\gamma \in \Gamma_{f(E)}$ has a maximal $f$-lifting in $A$ starting in $C$ (see [21, Corollary 3.3.II]). Let $\Gamma^{*}$ be a family of all maximal $f$-liftings of $\Gamma_{f(E)}$ starting in $C$. Note that $\Gamma^{*} \subset \Gamma_{E}$. Moreover, $\Gamma_{f(E)}>f\left(\Gamma^{*}\right)$ and, consequently,

$$
M\left(\Gamma_{f(E)}\right) \leq M\left(f\left(\Gamma^{*}\right)\right)
$$

Consider $S_{\varepsilon}=S\left(x_{0}, \varepsilon\right), S_{\varepsilon_{0}}=S\left(x_{0}, \varepsilon_{0}\right)$, with $\varepsilon_{0}$ satisfying the assumption of lemma and $\varepsilon \in\left(0, \varepsilon_{0}^{\prime}\right)$. Let $R\left(r_{1}, r_{2}, x_{0}\right)=\left\{x \in \mathbb{R}^{n}: r_{1}<\left|x-x_{0}\right|<r_{2}\right\}$. Note that 
$\Gamma\left(S_{\varepsilon}, S_{\varepsilon_{0}}, R\left(\varepsilon, \varepsilon_{0}, x_{0}\right)\right)<\Gamma^{*}$ and, consequently, $f\left(\Gamma\left(S_{\varepsilon}, S_{\varepsilon_{0}}, R\left(\varepsilon, \varepsilon_{0}, x_{0}\right)\right)\right)<f\left(\Gamma^{*}\right)$. Hence

$$
M\left(f\left(\Gamma^{*}\right)\right) \leq M\left(f\left(\Gamma\left(S_{\varepsilon}, S_{\varepsilon_{0}}, R\left(\varepsilon, \varepsilon_{0}, x_{0}\right)\right)\right)\right) .
$$

The relations (2.4) and (2.5) yield

$$
M\left(\Gamma_{f(E)}\right) \leq M\left(f\left(\Gamma\left(S_{\varepsilon}, S_{\varepsilon_{0}}, R\left(\varepsilon, \varepsilon_{0}, x_{0}\right)\right)\right)\right)
$$

and, consequently,

$$
\text { cap } f(E) \leq M\left(f\left(\Gamma\left(S_{\varepsilon}, S_{\varepsilon_{0}}, R\left(\varepsilon, \varepsilon_{0}, x_{0}\right)\right)\right)\right) \text {. }
$$

Consider a family of Lebesgue measurable functions $\eta_{\varepsilon}(t)=\psi_{\varepsilon}(t) / I\left(\varepsilon, \varepsilon_{0}\right), t \in\left(\varepsilon, \varepsilon_{0}\right)$. Given $\varepsilon \in\left(0, \varepsilon_{0}^{\prime}\right)$, we have $\int_{\varepsilon}^{\varepsilon_{0}} \eta_{\varepsilon}(t) d t=1$. By (1.1)

$$
\begin{gathered}
M\left(f\left(\Gamma\left(S_{\varepsilon}, S_{\varepsilon_{0}}, R\left(\varepsilon, \varepsilon_{0}, x_{0}\right)\right)\right)\right) \leq \\
\leq \frac{1}{I^{n}\left(\varepsilon, \varepsilon_{0}\right)} \int_{\varepsilon<\left|x-x_{0}\right|<\varepsilon_{0}} Q(x) \cdot \psi_{\varepsilon}^{n}\left(\left|x-x_{0}\right|\right) d m(x)
\end{gathered}
$$

for every $\varepsilon \in\left(0, \varepsilon_{0}^{\prime}\right)$. Now the inequality (2.3) directly follows from (2.1), (2.6) and (2.7).

A chordal diameter of a set $E \subset \overline{\mathbb{R}^{n}}$ is the quantity

$$
h(E)=\sup _{x, y \in E} h(x, y) .
$$

Denote by $\omega_{n-1}$ the area of the unit sphere $\mathbb{S}^{n-1}$ in $\mathbb{R}^{n}$.

Lemma 2.2. Let $f: D \rightarrow \mathbb{R}^{n}, n \geq 2$ be an open discrete ring $Q$-mapping at a point $x_{0} \in D, D^{\prime}:=f(D) \subset B(0, r)$ and $h\left(\overline{\mathbb{R}^{n}} \backslash B(0, r)\right) \geq \delta>0$. Suppose that there exist numbers $p \leq n, \varepsilon_{0} \in\left(0\right.$, dist $\left.\left(x_{0}, \partial D\right)\right), \varepsilon_{0}^{\prime} \in\left(0, \varepsilon_{0}\right)$ and nonnegative Lebesgue measurable functions $\psi_{\varepsilon}:\left(\varepsilon, \varepsilon_{0}\right) \rightarrow[0, \infty], \varepsilon \in\left(0, \varepsilon_{0}^{\prime}\right)$ such that

$$
\int_{\varepsilon<\left|x-x_{0}\right|<\varepsilon_{0}} Q(x) \cdot \psi_{\varepsilon}^{n}\left(\left|x-x_{0}\right|\right) d m(x) \leq K \cdot I^{p}\left(\varepsilon, \varepsilon_{0}\right) \quad \forall \varepsilon \in\left(0, \varepsilon_{0}^{\prime}\right),
$$

where the quantity $I\left(\varepsilon, \varepsilon_{0}\right)$ is defined by (2.2). Then

$$
h\left(f(x), f\left(x_{0}\right)\right) \leq \frac{\alpha_{n}}{\delta} \exp \left\{-\beta_{n} I^{\gamma_{n, p}}\left(\left|x-x_{0}\right|, \varepsilon_{0}\right)\right\}
$$

for every $x \in B\left(x_{0}, \varepsilon_{0}^{\prime}\right)$ with

$$
\alpha_{n}=2 \lambda_{n}^{2}, \quad \beta_{n}=\left(\frac{\omega_{n-1}}{K}\right)^{\frac{1}{n-1}}, \quad \gamma_{n, p}=1-\frac{p-1}{n-1}, \quad \lambda_{n} \in\left[4,2 e^{n-1}\right) .
$$

Proof. Choosing $\varepsilon \in\left(0, \varepsilon_{0}^{\prime}\right)$ and $F\left(\varepsilon, \varepsilon_{0}\right):=K \cdot I^{p}\left(\varepsilon, \varepsilon_{0}\right)$ one gets from (2.3) and (2.8)

$$
\operatorname{cap} f(E) \leq K \cdot I^{p-n}\left(\varepsilon, \varepsilon_{0}\right)
$$


Applying [23, Lemma 2.2] and taking into account that $f(A) \subset B(0, r)$, one obtains

$$
\operatorname{cap} f(E) \geq \frac{\omega_{n-1}}{\left\{\log \frac{2 \lambda_{n}^{2}}{h(f(C)) h\left(\overline{\mathbb{R}^{n}} \backslash B(0, r)\right)}\right\}^{n-1}}
$$

where $\lambda_{n} \in\left[4,2 e^{n-1}\right), \quad \lambda_{2}=4$ and $\lambda_{n}^{1 / n} \rightarrow e$ as $n \rightarrow \infty$ (cf. [29, (7.21) and Lemma 7.22]). Combining (2.11) and (2.12) with assumption $h\left(\overline{\mathbb{R}^{n}} \backslash B(0, r)\right) \geq \delta$, we have

$$
h(f(C)) \leq \frac{2 \lambda_{n}^{2}}{\delta} \exp \left\{-\left(\frac{\omega_{n-1}}{K}\right)^{\frac{1}{n-1}}\left(I\left(\varepsilon, \varepsilon_{0}\right)\right)^{\frac{n-p}{n-1}}\right\} .
$$

Letting $\alpha_{n}=2 \lambda_{n}^{2}, \quad \beta_{n}=\left(\frac{\omega_{n-1}}{K}\right)^{\frac{1}{n-1}}, \quad \gamma_{n, p}=1-\frac{p-1}{n-1}$,

$$
h(f(C)) \leq \frac{\alpha_{n}}{\delta} \exp \left\{-\beta_{n} I^{\gamma_{n, p}}\left(\varepsilon, \varepsilon_{0}\right)\right\}
$$

Pick arbitrary $x \in D$ such that $\left|x-x_{0}\right|=\varepsilon, 0<\varepsilon<\varepsilon_{0}^{\prime}$. Then $x \in \overline{B\left(x_{0}, \varepsilon\right)}$ and, moreover, $f(x) \in f\left(\overline{B\left(x_{0}, \varepsilon\right)}\right)=f(C)$. Thus, the inequality holds for any $\varepsilon \in\left(0, \varepsilon_{0}^{\prime}\right)$. Since $\varepsilon \in\left(0, \varepsilon_{0}^{\prime}\right)$ is arbitrary, the relation (2.9) is fulfilled in the whole ball $B\left(x_{0}, \varepsilon_{0}^{\prime}\right)$.

By the well known Liouville theorem, every conformal mapping of a domain $D$ of $\mathbb{R}^{n}, n \geq 3$, is a restriction of a Möbius transformations of a domain $D$ (see e.g. [7] and [21, Theorem 2.5.I]). For $n=2$, it is true when $D:=\overline{\mathbb{R}^{2}}$ (see, e.g., [18]). Inversely, a restriction of a Möbius transformation $U: \overline{\mathbb{R}^{n}} \rightarrow \overline{\mathbb{R}^{n}}$ of a domain $D \backslash$ $\left\{\infty, U^{-1}(\infty)\right\}$ is conformal mapping and, consequently, $M(U(\Gamma))=M(\Gamma)$ for every Möbius transformation $U: \overline{\mathbb{R}^{n}} \rightarrow \overline{\mathbb{R}^{n}}$ and any family of curves $\Gamma$ in $\overline{\mathbb{R}^{n}}$ (cf. [27, Theorem 8.1]).

The following two lemmas describe the local behavior of the classes of equicontinuous mappings obeying (1.1).

Lemma 2.3. Let $\mathfrak{F}_{Q}$ be a family of open discrete $Q$-mappings $f: D \rightarrow \overline{\mathbb{R}^{n}}$ at $x_{0} \in D, n \geq 2$. Suppose that $p<n, \varepsilon_{0} \in\left(0\right.$, dist $\left.\left(x_{0}, \partial D\right)\right), \varepsilon_{0}^{\prime} \in\left(0, \varepsilon_{0}\right)$ and $\psi$ : $\left(0, \varepsilon_{0}\right) \rightarrow[0, \infty]$ is a nonnegative Lebesgue measurable function satisfying (2.2) such that the estimate (2.8) holds for $\psi_{\varepsilon} \equiv \psi$ and $I\left(\varepsilon, \varepsilon_{0}\right) \rightarrow \infty$ as $\varepsilon \rightarrow 0$.

Then $\mathfrak{F}_{Q}$ is equicontinuous at $x_{0}$ if and only if there exist $\varepsilon_{i}=\varepsilon_{i}\left(x_{0}\right), i=1,2$, $0<\varepsilon_{1}<\varepsilon_{2}<\varepsilon_{0}$, such that the inequality

$$
h\left(f(x), f\left(x_{0}\right)\right) \leq \alpha_{n} \exp \left\{-\widetilde{\beta_{n}} I^{\gamma_{n, p}}\left(\left|x-x_{0}\right|, \varepsilon_{2}\right)\right\}
$$

holds for every $f \in \mathfrak{F}_{Q}$ and all $x \in B\left(x_{0}, \varepsilon_{1}\right)$, where $\widetilde{\beta_{n}}=\left(\frac{\omega_{n-1}}{2 K}\right)^{\frac{1}{n-1}}, \alpha_{n}$ and $\gamma_{n, p}$ are defined by (2.10).

Proof. Since $I\left(\varepsilon, \varepsilon_{0}\right) \rightarrow \infty$ as $\varepsilon \rightarrow 0$, the sufficient condition of this lemma follows from Lemma 2.2. Now we show that inequality (2.14) holds when $\mathfrak{F}_{Q}$ is equicontinuous at $x_{0}$. Given $\sigma>0$, there exists $\Delta=\Delta\left(\sigma, x_{0}\right)$ such that

$$
h\left(f(x), f\left(x_{0}\right)\right)<\sigma
$$


whenever $\left|x-x_{0}\right|<\Delta$. Pick $\Delta<\varepsilon_{0}$. Given a mapping $f \in \mathfrak{F}_{Q}$ and a point $x_{0} \in D$, there is a Möbius transformation $U: \overline{\mathbb{R}^{n}} \rightarrow \overline{\mathbb{R}^{n}}$ such that

$$
U\left(f\left(x_{0}\right)\right)=0, \quad h\left(U(f(x)), U\left(f\left(x_{0}\right)\right)\right)=h\left(f(x), f\left(x_{0}\right)\right)
$$

(see [27, Theorem 12.2]). Clearly, the composition $v:=U \circ f$ is ring $Q$-mapping at $x_{0}$. For sufficiently small $\sigma>0$ the relations (2.15) and (2.16) yield $|v(x)| \leq 1$ for some $\varepsilon_{2}=\varepsilon_{2}\left(x_{0}\right)$ and all $x \in B\left(x_{0}, \varepsilon_{2}\right)$.

Consider the mapping $g:=\left.v\right|_{B\left(x_{0}, \varepsilon_{2}\right)}$. Since $I\left(\varepsilon, \varepsilon_{0}\right) \rightarrow \infty$ as $\varepsilon \rightarrow 0$, we have that $0<I\left(\varepsilon, \varepsilon_{0}\right)<2 I\left(\varepsilon, \varepsilon_{2}\right)<\infty$ for all $\varepsilon \in\left(0, \varepsilon_{1}\right)$ and some $\varepsilon_{1} \in\left(0, \varepsilon_{2}\right)$ and therefore by (2.8) that

$$
\int_{\varepsilon<\left|x-x_{0}\right|<\varepsilon_{2}} Q(x) \cdot \psi^{n}\left(\left|x-x_{0}\right|\right) d m(x) \leq 2 K \cdot I^{p}\left(\varepsilon, \varepsilon_{2}\right) \quad \forall \varepsilon \in\left(0, \varepsilon_{1}\right) .
$$

Replacing $\varepsilon_{2}, \varepsilon_{1}$ and $2 K$ by $\varepsilon_{0}, \varepsilon_{0}^{\prime}$ and $K$, we get the inequality (2.8). Now applying Lemma 2.2 to the mapping $g$ with the conditions (2.16) completes the proof.

In what follows, we also need the following lemma.

Lemma 2.4. Let $\mathfrak{F}_{Q}$ be a family of open discrete mappings $f: D \rightarrow \overline{\mathbb{R}^{n}}, n \geq 2$, obeying (1.1) at a point $x_{0} \in D$. Suppose that there exists a number $\widetilde{\varepsilon_{0}} \in\left(0\right.$, dist $\left.\left(x_{0}, \partial D\right)\right)$ such that the following property holds: for every $\varepsilon_{0} \in\left(0, \widetilde{\varepsilon_{0}}\right)$ there exists $\varepsilon_{0}^{\prime} \in\left(0, \varepsilon_{0}\right)$ and a nonnegative Lebesgue measurable function $\psi:\left(0, \widetilde{\varepsilon_{0}}\right) \rightarrow[0, \infty]$ such that

$$
0<I\left(\varepsilon, \varepsilon_{0}\right):=\int_{\varepsilon}^{\varepsilon_{0}} \psi(t) d t<\infty \quad \forall \varepsilon \in\left(0, \varepsilon_{0}^{\prime}\right)
$$

and

$$
\int_{\varepsilon<\left|x-x_{0}\right|<\varepsilon_{0}} Q(x) \cdot \psi^{n}\left(\left|x-x_{0}\right|\right) d m(x) \leq K \cdot I^{p}\left(\varepsilon, \varepsilon_{0}\right) \quad \forall \varepsilon \in\left(0, \varepsilon_{0}^{\prime}\right) .
$$

If the family $\mathfrak{F}_{Q}$ is equicontinuous at $x_{0}$, then there are $\varepsilon_{i}=\varepsilon_{i}\left(x_{0}\right), i=1,2,0<\varepsilon_{1}<$ $\varepsilon_{2}<\varepsilon_{0}$, such that

$$
h\left(f(x), f\left(x_{0}\right)\right) \leq \alpha_{n} \exp \left\{-\beta_{n} I^{\gamma_{n, p}}\left(\left|x-x_{0}\right|, \varepsilon_{2}\right)\right\}
$$

for every $f \in \mathfrak{F}_{Q}$ and all $x \in B\left(x_{0}, \varepsilon_{1}\right)$ with $\alpha_{n}, \beta_{n}$ and $\gamma_{n, p}$ defined by (2.10).

Proof. If the family $\mathfrak{F}_{Q}$ is equicontinuous at the point $x_{0}$, then for every $\sigma>0$ there exists $\Delta=\Delta\left(\sigma, x_{0}\right)$ such that (2.15) holds for every $x \in B\left(x_{0}, \Delta\right)$. Given a mapping $f \in \mathfrak{F}_{Q}$, there is a Möbius transformation $U: \overline{\mathbb{R}^{n}} \rightarrow \overline{\mathbb{R}^{n}}$ provided (2.16). The mapping $v:=U \circ f$ is a ring $Q$-mapping at $x_{0}$. For sufficiently small $\sigma>0$ the relations (2.15) and (2.16) yield $|v(x)| \leq 1$ for small enough $\sigma$, for some $\varepsilon_{2}=\varepsilon_{2}\left(x_{0}\right)$ and all $x \in B\left(x_{0}, \varepsilon_{2}\right)$. Consider the mapping $g:=\left.f\right|_{B\left(x_{0}, \varepsilon_{2}\right)}$. By (2.18) the double inequality $0<I\left(\varepsilon, \varepsilon_{2}\right)<\infty$ holds for all $\varepsilon \in\left(0, \varepsilon_{1}\right)$ and some $\varepsilon_{1} \in\left(0, \varepsilon_{2}\right)$ and therefore by (2.19)

$$
\int_{\varepsilon<\left|x-x_{0}\right|<\varepsilon_{2}} Q(x) \cdot \psi^{n}\left(\left|x-x_{0}\right|\right) d m(x) \leq K \cdot I^{p}\left(\varepsilon, \varepsilon_{2}\right) \quad \forall \varepsilon \in\left(0, \varepsilon_{1}\right) .
$$


Again applying Lemma 2.2 to the mapping $g$ with the conditions (2.16) completes the proof.

Remark 2.1. The inequalities (2.14) and (2.20) in Lemmas 2.3 and 2.4 are both of the same type and provide the upper bounds for the chordal distances between $f(x)$ and $f\left(x_{0}\right)$. The constant $\beta_{n}=\left(\frac{\omega_{n-1}}{K}\right)^{1 /(n-1)}$ in (2.20) is better than the corresponding constant $\widetilde{\beta_{n}}=\left(\frac{\omega_{n-1}}{2 K}\right)^{1 /(n-1)}$ in (2.14). On the other hand, the conditions (2.2), (2.8) of Lemma 2.3 hold for one fixed $\varepsilon_{0}$, while the assertion of Lemma 2.4 is fulfilled for infinitely many $\varepsilon_{0}$ with the same $K>0$. Applications of both lemmas will be presented in the next section.

\section{On necessary and sufficient conditions of equicon- tinuity}

We start to prove Theorem 1.1 by applying Lemma 2.3 for an appropriate function $\psi$. Letting $\psi(t):=\frac{1}{t \log 1 / t}$ and $\varepsilon_{0}<1 / e$, we have that

$$
I\left(\varepsilon, \varepsilon_{0}\right)=\int_{\varepsilon}^{\varepsilon_{0}} \psi(t) d t=\log \frac{\log \frac{1}{\varepsilon}}{\log \frac{1}{\varepsilon_{0}}}
$$

where $I\left(\varepsilon, \varepsilon_{0}\right) \rightarrow \infty$ as $\varepsilon \rightarrow 0$ and the condition (2.8) holds by [8, Corollary 2.3] (cf. [15, Lemma 6.1]). Thus, the condition (1.3) follows from (2.9) for the chosen function $\psi . \square$.

Given a Lebesgue measurable function $Q: D \rightarrow[0, \infty]$, denote by $q_{x_{0}}(r)$ the integral average of $Q(x)$ over the sphere $\left|x-x_{0}\right|=r$,

$$
q_{x_{0}}(r):=\frac{1}{\omega_{n-1} r^{n-1}} \int_{\left|x-x_{0}\right|=r} Q(x) d S,
$$

where $d S$ denotes an element of the sphere area. The following statements are relied on Lemma 2.4

Theorem 3.1. Given $x_{0} \in D$ and a Lebesgue measurable function $Q: D \rightarrow[0, \infty]$, suppose that the condition (1.4) holds for some $\varepsilon_{0}=\varepsilon_{0}\left(x_{0}\right) \in\left(0\right.$, dist $\left.\left(x_{0}, \partial D\right)\right)$. A family of open discrete ring $Q$-mappings $f: D \rightarrow \overline{\mathbb{R}^{n}}$ at the point $x_{0} \in D$ is equicontinuous at $x_{0}$ if and only if the inequality

$$
h\left(f(x), f\left(x_{0}\right)\right) \leq \alpha_{n} \cdot \exp \left\{-\int_{\left|x-x_{0}\right|}^{\varepsilon_{0}} \frac{d t}{t q_{x_{0}}^{\frac{1}{n-1}}(t)}\right\}
$$

holds for all $x \in B\left(x_{0}, \varepsilon_{0}^{\prime}\right)$ and some $\varepsilon_{0}^{\prime} \in\left(0, \varepsilon_{0}\right)$ with $\alpha_{n}$ defined in Lemma 2.2 .

Proof. The sufficient condition of Theorem 3.1 is established by a direct application of the inequality (3.3) and the condition (1.4) from Theorem 1.2, So, it remains to 
prove the necessity. To this end, pick

$$
\psi(t)=\left\{\begin{array}{rlrl}
1 /\left[t q_{x_{0}}^{\frac{1}{n-1}}(t)\right], & & t \in\left(\varepsilon, \varepsilon_{0}\right), \\
0, & t \notin\left(\varepsilon, \varepsilon_{0}\right)
\end{array}\right.
$$

By (1.4), for sufficiently small $\varepsilon_{0}>0$ there exists $\varepsilon_{0}^{\prime} \in\left(0, \varepsilon_{0}\right)$ such that $I\left(\varepsilon, \varepsilon_{0}\right):=$ $\int_{\varepsilon}^{\varepsilon_{0}} \psi(t) d t>0$ for every $\varepsilon \in\left(0, \varepsilon_{0}^{\prime}\right)$, and, moreover, $I\left(\varepsilon, \varepsilon_{0}\right)<\infty$ for every $\varepsilon \in\left(0, \varepsilon_{0}\right)$ (see [26, Remark 3]). A direct calculation yields

$$
\int_{\varepsilon<\left|x-x_{0}\right|<\varepsilon_{0}} Q(x) \cdot \psi^{n}\left(\left|x-x_{0}\right|\right) d m(x)=\omega_{n-1} \cdot I\left(\varepsilon, \varepsilon_{0}\right) .
$$

Thus, $\psi$ satisfies condition (2.19) with $p=1$ and $K=\omega_{n-1}$, and the desired conclusion follows from Lemma 2.4 .

The following statement follows directly from Theorem 3.1 .

Corollary 3.1. Given $x_{0} \in D$ and a Lebesgue measurable function $Q: D \rightarrow[0, \infty]$, assume that the estimate

$$
q_{x_{0}}(r) \leq C\left(\log \frac{1}{r}\right)^{n-1} \quad \forall r \in\left(0, \varepsilon_{0}\right)
$$

holds for some $\varepsilon_{0}=\varepsilon\left(x_{0}\right) \in\left(0\right.$, dist $\left.\left(x_{0}, \partial D\right)\right)$. Then a family of all open discrete ring $Q$-mappings $f: D \rightarrow \overline{\mathbb{R}^{n}}$ at $x_{0}$ is equicontinuous at $x_{0}$ if and only if

$$
h\left(f(x), f\left(x_{0}\right)\right) \leq \frac{M}{\log \left(\frac{1}{C}\right)^{\frac{1}{n-1}} \frac{1}{\sqrt{x-x_{0}} \mid}} \quad \forall x \in B\left(x_{0}, \varepsilon_{0}^{\prime}\right)
$$

and for some $\varepsilon_{0}^{\prime} \in\left(0, \varepsilon_{0}\right)$, where the constant $M$ depends only on $n$ and $x_{0}$.

The next statement shows that the condition (1.4) in Theorem (3.1) cannot be removed.

Theorem 3.2. Let $D$ be a domain in $\mathbb{R}^{n}, n \geq 2, x_{0} \in D$, and $0<\varepsilon_{0}<$ $\operatorname{dist}\left(x_{0}, \partial D\right)$. Given a function $Q: D \rightarrow[1, \infty], Q \in L_{\text {loc }}^{1}(D)$, with

$$
\int_{0}^{\varepsilon_{0}} \frac{d t}{t q_{x_{0}}^{\frac{1}{n-1}}(t)}<\infty
$$

where $q_{x_{0}}$ is defined in (3.2). Then there exists a family of uniformly bounded ring $Q$-mappings at the point $x_{0}$ which is not equicontinuous at $x_{0}$.

Proof. Without loss of generality, we may assume that $D=\mathbb{B}^{n}=\left\{x \in \mathbb{R}^{n}:|x|<1\right\}$ and $x_{0}=0$. Define a sequence of mappings $f_{m}: \mathbb{B}^{n} \rightarrow \mathbb{R}^{n}$ by

$$
f_{m}(x)=\frac{x}{|x|} \rho_{m}(|x|), \quad f_{m}(0):=0
$$

where

$$
\rho_{m}(r)=\exp \left\{-\int_{r}^{1} \frac{d t}{t q_{0, m}^{1 /(n-1)}(t)}\right\}, \quad q_{0, m}(r):=\frac{1}{\omega_{n-1} r^{n-1}} \int_{|x|=r} Q_{m}(x) d S,
$$




$$
Q_{m}(x)=\left\{\begin{array}{cc}
Q(x), & |x|>1 / m \\
1, & |x| \leq 1 / m
\end{array}\right.
$$

Now we show that every $f_{m}, m=1,2, \ldots$, is a ring $Q$-homeomorphism at the point $x_{0}=0$. Obviously, $f(S(0, r))=S\left(0, R_{m}\right)$ where

$$
R_{m}=\exp \left\{-\int_{r}^{1} \frac{d t}{t q_{0, m}^{1 /(n-1)}(t)}\right\}, \quad r \in(0,1) .
$$

Note that

$$
f_{m}\left(\Gamma\left(S\left(0, r_{1}\right), S\left(0, r_{2}\right), R\left(r_{1}, r_{2}, 0\right)\right)\right)=\Gamma\left(S\left(0, R_{1, m}\right), S\left(0, R_{2, m}\right), R\left(R_{1, m}, R_{2, m}, 0\right)\right)
$$

where $R_{i, m}=\exp \left\{-\int_{r_{i}}^{1} \frac{d t}{t q_{0, m}^{1 /(m-1)}(t)}\right\}, i=1,2$. Following to [27, 7.5],

$$
M\left(f\left(\Gamma\left(S\left(0, r_{1}\right), S\left(0, r_{2}\right), R\left(r_{1}, r_{2}, 0\right)\right)\right)\right)=\frac{\omega_{n-1}}{\left(\int_{r_{1}}^{r_{2}} \frac{d t}{t q_{0, m}^{1 /(n-1)}(t)}\right)^{n-1}} \leq \frac{\omega_{n-1}}{\left(\int_{r_{1}}^{r_{2}} \frac{d t}{t q_{0}^{1 /(n-1)}(t)}\right)^{n-1}},
$$

where $q_{0}(r)$ is defined by (3.2) at the origin. Consequently, the mapping $f_{m}$ is a ring $Q$-mapping at 0 (see [25, Theorem 1]). Since $\left|f_{m}(x)\right| \leq 1$ for every $m \in \mathbb{N}$, the family $\left\{f_{m}(x)\right\}_{m=1}^{\infty}$ is uniformly bounded. On the other hand, for every sequence $x_{m}$ provided $\left|x_{m}\right|=1 / m, m=1,2, \ldots$, one obtains $\left|f_{m}\left(x_{m}\right)\right| \geq \sigma$, where $\sigma>0$ does not depend on $m$. Thus, the family $\left\{f_{m}(x)\right\}_{m=1}^{\infty}$ is not equicontinuous at the origin.

\section{On equicontinuity of map families omitting sets}

The main results of this section are relied on Vuorinen's approach in [28]. Here we prove more general results on equicontinuity of open discrete mappings satisfying the estimates (1.1)-(1.2) (cf. [23]). Following [28], we establish these results on equicontinuity of the mapping families involving set function of a special kind.

Let $B^{*}(x, t)=\left\{y \in \overline{\mathbb{R}^{n}}: h(x, y)<t\right\}$ be a spherical ball centered at the point $x$ of the radius $t$. Given $x \in \overline{\mathbb{R}^{n}}, E \subset \overline{\mathbb{R}^{n}}$ and $0<r<t<1$, we define

$$
\left\{\begin{array}{r}
m_{t}(E, r, x)=M\left(\Gamma\left(\partial B^{*}(x, t), E \cap \overline{B^{*}(x, r)}\right)\right) \\
m(E, x)=m_{\sqrt{3} / 2}\left(E, \frac{\sqrt{2}}{2}, x\right)
\end{array}\right.
$$

and

$$
\left\{\begin{array}{r}
c(E, x)=\max \{m(E, x), m(E, \widetilde{x})\}, \\
c(E)=\inf _{x \in \overline{\mathbb{R}^{n}}} c(E, x),
\end{array}\right.
$$

where $\widetilde{x}=-x /|x|^{2}$. Given a compact set $E \subset \overline{\mathbb{R}^{n}}$, we have $c(E)=0$ if and only if cap $E=0$ (see [28, Corollary 3.19]).

Let $D$ be a domain in $\mathbb{R}^{n}, n \geq 2$. Denote by $\mathfrak{F}_{Q, \delta}$ a family of all open discrete ring $Q$-mappings $f: D \rightarrow \overline{\mathbb{R}^{n}} \backslash E_{f}$ at $x_{0} \in D$ with $c\left(E_{f}\right) \geq \delta>0$, where $E_{f}$ is compact and $c(\cdot)$ is defined by (4.1). 
The following auxiliary result is of independent interest.

Lemma 4.1. Suppose that there exist $\varepsilon_{0}>0, \varepsilon_{0}<\operatorname{dist}\left(x_{0}, \partial D\right), \varepsilon_{1} \in\left(0, \varepsilon_{0}\right)$ and a function $\psi:\left(0, \varepsilon_{0}\right) \rightarrow[0, \infty]$ with

$$
0<I\left(\varepsilon, \varepsilon_{0}\right)=\int_{\varepsilon}^{\varepsilon_{0}} \psi(t) d t<\infty \quad \forall \varepsilon \in\left(0, \varepsilon_{1}\right)
$$

such that

$$
\int_{\varepsilon<\left|x-x_{0}\right|<\varepsilon_{0}} Q(x) \cdot \psi^{n}\left(\left|x-x_{0}\right|\right) d m(x)=o\left(I^{n}\left(\varepsilon, \varepsilon_{0}\right)\right)
$$

as $\varepsilon \rightarrow 0$. Then the family $\mathfrak{F}_{Q, \delta}$ is equicontinuous at $x_{0}$.

Proof. Given a mapping $f \in \mathfrak{F}_{Q, \delta}$, consider the condensers $E=(A, C)$ and $E^{\prime}=f(E)=(f(A), f(C))$ where $C:=\overline{B\left(x_{0}, \varepsilon\right)}, \varepsilon \in\left(0, \varepsilon_{1}\right), A=B\left(x_{0}, r_{0}\right), r_{0}=$ $\operatorname{dist}\left(x_{0}, \partial D\right)$. Following the notation $\Gamma_{E}$ in the proof of Lemma 2.1. let $\Gamma_{E^{\prime}}$ be a corresponding curve family for the condenser $E^{\prime}$. Note that by [10, Theorem 1.I.46] $\Gamma\left(f(C), E_{f}\right)>\Gamma_{E^{\prime}}$ and, consequently, by [27, Theorem 6.4]), Lemma 2.1] and (4.3), one gets

$$
M\left(\Gamma\left(f(C), E_{f}\right)\right) \leq M\left(\Gamma_{E^{\prime}}\right)=\operatorname{cap} f(E) \leq \alpha(\varepsilon)
$$

where $\alpha(\varepsilon) \rightarrow 0$ as $\varepsilon \rightarrow 0$. On the other hand, by [28, Theorem 3.14],

$$
M\left(\Gamma\left(f(C), E_{f}\right)\right) \geq \beta_{n} \min \left\{c(f(C)), c\left(E_{f}\right)\right\}
$$

where the constant $\beta_{n}$ depends only on $n$.

Since $c(F) \geq a_{n} h(F)$ for every connected set $F$ in $\overline{\mathbb{R}^{n}}$,

$$
c(f(C)) \geq a_{n} \cdot h(f(C))
$$

where $h(F)$ is the chordal diameter of $F$ and $a_{n}$ is some constant (see [28, Corollary 3.13]). It is known that $c(E) \leq \omega_{n-1} \cdot(\log \sqrt{3})^{1-n}$ for every set $E \subset \overline{\mathbb{R}^{n}}$, consequently,

$$
\frac{c(E)}{\omega_{n-1} \cdot(\log \sqrt{3})^{1-n}} \leq 1,
$$

cf. [28, (3.7)] and (4.1). If the minimum in (4.5) equals $c(f(C))$, then we have by (4.6) and (4.7)

$$
M\left(\Gamma\left(f(C), E_{f}\right)\right) \geq \beta_{n} \cdot a_{n} \cdot h(f(C)) \geq \frac{\beta_{n} \cdot a_{n} \cdot h(f(C)) c\left(E_{f}\right)}{\omega_{n-1} \cdot(\log \sqrt{3})^{1-n}} .
$$

For the case when $\min \left\{c(f(C)), c\left(E_{f}\right)\right\}=c\left(E_{f}\right)$, the inequality (4.5) yields

$$
M\left(\Gamma\left(f(C), E_{f}\right)\right) \geq \beta_{n} \cdot c\left(E_{f}\right) \geq \beta_{n} \cdot h(f(C)) c\left(E_{f}\right) .
$$

Letting $c_{n}:=\min \left\{\beta_{n}, \frac{\beta_{n} \cdot a_{n}}{\omega_{n-1} \cdot(\log \sqrt{3})^{1-n}}\right\}$, one can derive from (4.8) and (4.9) that

$$
M\left(\Gamma\left(f(C), E_{f}\right)\right) \geq c_{n} \cdot h(f(C)) c\left(E_{f}\right) \geq c_{n} \cdot \delta \cdot h(f(C))
$$


and combining (4.4) and (4.10) the following estimate holds

$$
h(f(C)) \leq \frac{\alpha(\varepsilon)}{c_{n} \delta}
$$

Since $\alpha(\varepsilon) \rightarrow 0$ as $\varepsilon \rightarrow 0$, one can conclude from (4.11) that for every $\sigma>0$ there exists $\Delta=\Delta(\sigma)$ such that $h(f(C))<\sigma$ whenever $\varepsilon<\Delta$. In other words, $h\left(f(x), f\left(x_{0}\right)\right)<\sigma$ for all $x \in B\left(x_{0}, \varepsilon\right), \varepsilon<\Delta$. Hence, the inequality $h\left(f(x), f\left(x_{0}\right)\right)<\sigma$ holds for all $x \in B\left(x_{0}, \Delta\right)$ and every $f \in \mathfrak{F}_{Q, \Delta}$, i.e. the family $\mathfrak{F}_{Q, \Delta}$ is equicontinuous at the point $x_{0}$.

The following result is a strengthen version of [26, Lemma 8].

Lemma 4.2. Let $Q: D \rightarrow[0, \infty]$ be a Lebesgue measurable function. Assume that either of the following conditions holds at $\left.x_{0} \in D: 1\right) Q \in F M O\left(x_{0}\right)$; 2) $q_{x_{0}}(r) \leq$ $\left.C \cdot\left(\log \frac{1}{r}\right)^{n-1} ; 3\right)$ the relation (1.4) is true for some $\varepsilon_{0}=\varepsilon_{0}\left(x_{0}\right)$. Then the conditions (4.2)-(4.3) of Lemma 4.1 hold.

Proof. We start with the assumption 1) choosing $\psi(t):=\frac{1}{t \log 1 / t}$ and $\varepsilon_{0}<e^{-1}$. Then the conditions (3.1) and (4.3) hold (see [15, Lemma 6.1]). Thus, the relations (4.2)-(4.3) are fulfilled.

Since the assumption 2) is a particular case of 3), we should prove the implication $(1.4) \Rightarrow(4.2)-(4.3)$. Given sufficiently small $\varepsilon>0$, pick $\psi$ by (3.4). In accordance to (1.4), there exists $\varepsilon_{1} \in\left(0, \varepsilon_{0}\right)$ provided $I\left(\varepsilon, \varepsilon_{0}\right)>0$ for all $\varepsilon \in\left(0, \varepsilon_{1}\right)$. Note that $I\left(\varepsilon, \varepsilon_{0}\right)<\infty$ for every $\varepsilon \in\left(0, \varepsilon_{0}\right)$ (see [26, Remark 3]). Now arguing similar to the proof of Theorem 3.1, one obtains equality (3.5) and, moreover, the asymptotic relation (4.3).

The assertion of Theorem 1.2 directly follows from Lemma 4.1 and Lemma 4.2 .

\section{Applications and related results}

Now we present some important particular cases of Theorem 1.2. The first one relates to the case $Q(x)$ is uniformly bounded by a constant $K$, i.e., $Q(x) \leq K=$ const and $E_{f}=E \subset \overline{\mathbb{R}^{n}}$. Thus, we recall the well-known results on equicontinuity for quasiregular mappings (see [13, Theorem 3.17] and [21, Corollary 2.7.III]).

Corollary 5.1. Given a compact set $E \subset \overline{\mathbb{R}^{n}}$ with cap $E>0$, the family of all $K$-quasiregular mappings $f: D \rightarrow \overline{\mathbb{R}^{n}} \backslash E$ is equicontinuous at every point $x_{0} \in D$.

As a consequence for $E_{f} \equiv E \subset \overline{\mathbb{R}^{n}}$, we obtain the following author result (see [23, Theorems 5.1-5.2]).

Corollary 5.2. Given a compact set $E \subset \overline{\mathbb{R}^{n}}$ with cap $E>0$, a family of all open discrete $Q$-mappings $f: D \rightarrow \overline{\mathbb{R}^{n}} \backslash E$ is equicontinuous at every point $x_{0} \in D$ if either of the conditions 1)-3) of Theorem [1.2 holds.

The following result is based on Theorems 1.1, 1.2, 3.1 and Corollary 3.1. 
Theorem 5.1. Let $D$ be a domain in $\mathbb{R}^{n}, n \geq 2$. Denote by $\mathfrak{F}_{Q, \delta}$ a family of all open discrete ring $Q$-mappings $f: D \rightarrow \overline{\mathbb{R}^{n}} \backslash E_{f}$ at $x_{0} \in D$ with $c\left(E_{f}\right) \geq \delta>0$, where $E_{f}$ is compact and $c(\cdot)$ is defined by (4.1). Then:

1. Every $f \in \mathfrak{F}_{Q, \delta}$ satisfies the estimate (1.3) for all $x \in B\left(x_{0}, \varepsilon_{0}\left(x_{0}\right)\right)$ and some $\varepsilon_{0}\left(x_{0}\right) \in\left(0\right.$, dist $\left.\left(x_{0}, \partial D\right)\right)$ whenever $Q \in F M O\left(x_{0}\right)$ where $p=p(n, Q)>0$ and $C_{n}>0$ are some constants. и всех

2. Every $f \in \mathfrak{F}_{Q, \delta}$ satisfies the estimate (3.3) for some constant $\alpha_{n}>0$ whenever the condition (1.4) holds for some $\varepsilon_{0} \in\left(0\right.$, dist $\left.\left(x_{0}, \partial D\right)\right)$

3. Every $f \in \mathfrak{F}_{Q, \delta}$ satisfies the estimate (3.7) in a neighborhood of the point $x_{0}$ whenever the inequality (3.6) holds with $C>0$ where $M>0$ is some constant depending only on $n$ and point $x_{0}$.

Finally, we give important applications of our main results to the Sobolev classes. Let $x \in D$ be a point of differentiability of $f$. Set

$$
l\left(f^{\prime}(x)\right)=\min _{h \in \mathbb{R}^{n} \backslash\{0\}} \frac{\left|f^{\prime}(x) h\right|}{|h|},\left\|f^{\prime}(x)\right\|=\max _{h \in \mathbb{R}^{n} \backslash\{0\}} \frac{\left|f^{\prime}(x) h\right|}{|h|}, J(x, f)=\operatorname{det} f^{\prime}(x),
$$

and define for such $x \in D$ the inner dilatation of $f$ at $x$ by

$$
K_{I}(x, f)=\left\{\begin{array}{rr}
\frac{|J(x, f)|}{l\left(f^{\prime}(x)\right)^{n},} & J(x, f) \neq 0, \\
1, & f^{\prime}(x)=0, \\
\infty, & \text { otherwise }
\end{array}\right.
$$

Recall that a point $y_{0} \in D$ is said to be a branch point of a mapping $f: D \rightarrow \mathbb{R}^{n}$ if, for every neighborhood $U$ of $y_{0}$, a restriction $\left.f\right|_{U}$ fails to be homeomorphic. A set of all branch points of $f$ is denoted by $B_{f}$.

The following statement follows from [24, Corollary 2] and Theorems 1.1, 3.1 and Corollary 3.1 as well. Let $\mathfrak{A}_{Q}$ be a family of all discrete open mappings $f: D \rightarrow \mathbb{R}^{n}$ of Sobolev's class $W_{l o c}^{1, n}$ with a branch set $B_{f}$ of the Lebesgue measure zero. Assume that $K_{I}(x, f) \leq Q(x) \in L_{l o c}^{1}$. Then we have:

Corollary 5.3. 1. Every $f \in \mathfrak{A}_{Q}$ satisfies the estimate (1.3) for all $x \in$ $B\left(x_{0}, \varepsilon_{0}\left(x_{0}\right)\right)$ and some $\varepsilon_{0}\left(x_{0}\right) \in\left(0\right.$, dist $\left.\left(x_{0}, \partial D\right)\right)$ whenever $Q \in F M O\left(x_{0}\right)$ where $p=p(n, Q)>0$ and $C_{n}>0$ are some constants.

2. Every $f \in \mathfrak{A}_{Q}$ satisfies the estimate (3.3) with some constant $\alpha_{n}>0$ whenever the condition (1.4) holds for some $\varepsilon_{0} \in\left(0\right.$, dist $\left.\left(x_{0}, \partial D\right)\right)$.

3. Every $f \in \mathfrak{A}_{Q}$ satisfies the estimate (3.7) in a neighborhood of the point $x_{0}$ whenever the inequality (3.6) holds with $C>0$ where $M>0$ is some constant depending only on $n$ and point $x_{0}$.

Denote by $\mathfrak{B}_{Q, \delta}$ a family of all discrete open mappings $f: D \rightarrow \mathbb{R}^{n} \backslash E_{f}$ of $W_{l o c}^{1, n}$ with a branch set $B_{f}$ of the Lebesgue measure zero such that $K_{I}(x, f) \leq Q(x) \in L_{l o c}^{1}$ and $c\left(E_{f}\right) \geq \delta>0$. The following statement follows from [24, Corollary 2] and Theorems 1.1, 3.1, and Corollary 3.1. 
Corollary 5.4. A family of the mappings $\mathfrak{B}_{Q, \delta}$ is equicontinuous at $x_{0}$ provided that either of the following conditions 1)-3) of Theorem 1.2 holds.

The modulus and capacity inequalities provide one of the main tools in investigation the basic properties of a wide spectrum of mappings (see, e.g., [1]-[5], [8], [11], [12][17], 19], 21]-[29]). Thus, the results of the paper can be successfully applied to these classes of mappings.

\section{References}

[1] Andreian Cazacu C., On the length-area dilatation, Complex Var. Theory Appl. 50 (2005), no. 7-11, 765-776.

[2] Bishop C.J., Gutlyanskit V.Ya., Martio O., Vuorinen M., On conformal dilatation in space, Intern. Journ. Math. and Math. Sci. 22 (2003), 1397-1420.

[3] Cristea M., Local homeomorphisms having local $A C L^{n}$ inverses, Compl. Var. and Ell. Eq. 53 (2008), no. 1, 77-99.

[4] Golberg A., Differential properties of $(\alpha, Q)$ - homeomorphisms, Further Progress in Analysis, World Scientific Publ. (2009), 218-228.

[5] Golberg A., Salimov R., Topological mappings of integrally bounded $p-$ moduli, Ann. Univ. Bucharest (math. series) 3 (2012), no. 1, 49-66.

[6] Gutlyanskit V.Ya., Ryazanov V.I., Srebro U., Yakubov E., The Beltrami Equation: A Geometric Approach, Developments in Mathematics, vol. 26, Springer, New York etc., 2012.

[7] Hartman P., On isometries and on a theorem of Liouville, Math. Z. 69 (1958), 202-210.

[8] Ignat'ev A. And Ryazanov V., Finite mean oscillation in the mapping theory, Ukr. Math. Bull. 2 (2005), no. 3, 403--424.

[9] John F., Nirenberg L., On functions of bounded mean oscillation, Comm. Pure Appl. Math. 14 (1961), 415-426.

[10] Kuratowski K., Topology, Vol. 2., Academic Press, New York and London, 1968.

[11] Lehto O. and Virtanen K., Quasiconformal Mappings in the Plane, Springer, New York etc., 1973.

[12] Martio O., Rickman S., VÄısälä J., Definitions for quasiregular mappings, Ann. Acad. Sci. Fenn. Ser. A I. Math. 448 (1969), 1-40.

[13] Martio O., Rickman S. and VÄISÄLÄ J., Distortion and singularities of quasiregular mappings, Ann. Acad. Sci. Fenn. Ser. A1. 465 (1970), 1-13. 
[14] Martio O., Rickman S., VÄIsÄlä J., Topological and metric properties of quasiregular mappings, Ann. Acad. Sci. Fenn. Ser. A I. Math. 488 (1971), 1-31.

[15] Martio O., Ryazanov V., Srebro U. and Yakubov E., Moduli in Modern Mapping Theory, Springer Science + Business Media, New York: LLC, 2009.

[16] Miklyukov V.M., Conformal Maps of Nonsmooth Surfaces and Their Applications, Exlibris Corporation, Philadelphia, 2008.

[17] Miniowitz R., Normal families of quasimeromorphic mappings, Proc. Amer. Math. Soc. 84 (1982), no. 1, 35-43.

[18] Mostow G.D., Quasi-conformal mappings in $n$-space and the rigidity of hyperbolic space forms, Inst. Hautes Études Sci. Publ. Math. 34 (1968), 53-104.

[19] Poletskin E.A., The modulus method for non-homeomorphic quasiconformal mappings, Mat. Sb. 83 (125) (1970), no. 2, 261-272.

[20] Reshetnyak Yu.G., Space Mappings with Bounded Distortion, Transl. of Math. Monographs 73, AMS, 1989.

[21] Rickman S., Quasiregular Mappings, Springer, Berlin etc., 1993.

[22] Ryazanov V., Srebro U. and Yakubov, On ring solutions of Beltrami equations, J. Anal. Math. 96 (2005), 117-150.

[23] Sevost'yanov E.A., Theory of moduli, capacities and normal families of mappings admitting a branching, Ukr. Math. Bull. 4 (2007), no. 4, 573-593.

[24] Sevost'yanov E.A., Generalization of one Poletskii lemma to classes of space mappings, Ukrainian Math. J. 61 (2009), no. 7, 1151-1157.

[25] Sevost'yanov E.A., On the integral characterization of some generalized quasiregular mappings and the significance of the conditions of divergence of integrals in the geometric theory of function, Ukrainian Math. J. 61 (2009), no. 10, $1610-1623$.

[26] Sevost'yanov E.A., On the Branch Points of Mappings with the Unbounded Coefficient of Quasiconformality, Siberian Math. J. 51 (2010), no. 5, 899-912.

[27] VÄIsÄLÄ J., Lectures on n-Dimensional Quasiconformal Mappings, Lecture Notes in Math. 229, Berlin etc., Springer-Verlag, 1971.

[28] Vuorinen M., Some inequalities for the moduli of curve families, Michigan Math. J. 30 (1983), 369-380.

[29] Vuorinen M., Conformal Geometry and Quasiregular Mappings, Lecture Notes in Math. 1319, Berlin etc., Springer-Verlag, 1988.

[30] Zalcman L., A heuristic principle in complex function theory, Amer. Math. Monthly 82 (1975), no. 8, 813--817. 


\section{Evgeny Sevost'yanov:}

Institute of Applied Mathematics and Mechanics,

National Academy of Sciences of Ukraine,

74 Roze Luxemburg Str., Donetsk,

83114, UKRAINE

e-mail: brusin2006@rambler.ru 